\title{
New search for processes violating the Pauli exclusion principle in sodium and in iodine
}

\author{
R. Bernabei ${ }^{1,2, a}$, P. Belli ${ }^{2}$, F. Cappella ${ }^{3,4}$, R. Cerulli ${ }^{5}$, C.J. Dai ${ }^{6}$, A. d'Angelo ${ }^{3,4}$, H.L. He ${ }^{6}$, A. Incicchitti ${ }^{4}$, \\ H.H. Kuang ${ }^{6}$, X.H. Ma ${ }^{6}$, F. Montecchia ${ }^{1,2}$, F. Nozzoli ${ }^{1,2}$, D. Prosperi ${ }^{3,4}$, X.D. Sheng ${ }^{6}$, Z.P. Ye Ye,7 $^{6,7}$ \\ ${ }^{1}$ Dip. di Fisica, Università di Roma “Tor Vergata”, 00133 Rome, Italy \\ ${ }^{2}$ INFN, sez. Roma "Tor Vergata", 00133 Rome, Italy \\ ${ }^{3}$ Dip. di Fisica, Università di Roma "La Sapienza", 00185 Rome, Italy \\ ${ }^{4}$ INFN, sez. Roma, 00185 Rome, Italy \\ ${ }^{5}$ Laboratori Nazionali del Gran Sasso, INFN, Assergi, Italy \\ ${ }^{6}$ IHEP, Chinese Academy, P.O. Box 918/3, Beijing 100039, China \\ ${ }^{7}$ University of Jing Gangshan, Jiangxi, China
}

Received: 3 March 2009 / Revised: 27 April 2009 / Published online: 4 June 2009

(c) Springer-Verlag / Società Italiana di Fisica 2009

\begin{abstract}
In this paper a new search for non-Paulian nuclear processes, i.e. processes normally forbidden by the Pauli Exclusion Principle (PEP), is presented. It has been carried out at the Gran Sasso National Laboratory of the INFN by means of the highly radiopure DAMA/LIBRA set-up (sensitive mass of about $250 \mathrm{~kg}$ highly radiopure $\mathrm{NaI}(\mathrm{Tl})$ ). In particular, a new improved upper limit for the spontaneous non-Paulian emission rate of protons with energy $E_{p} \geq 10 \mathrm{MeV}$ in ${ }^{23} \mathrm{Na}$ and ${ }^{127} \mathrm{I}$ has been obtained: $1.63 \times 10^{-33} \mathrm{~s}^{-1}$ (90\% C.L.). The corresponding limit on the relative strength $\left(\delta^{2}\right)$ for the searched non-Paulian transition is $\delta^{2} \lesssim(3-4) \times 10^{-55}$ (90\% C.L.). Moreover, PEP-violating electron transitions in iodine atoms have also been investigated. Lifetimes shorter than $4.7 \times 10^{30} \mathrm{~s}$ are excluded at $90 \%$ C.L.; this allows us to derive the limit $\delta_{e}^{2}<1.28 \times 10^{-47}(90 \%$ C.L.). This latter limit can also be related to a possible finite size of the electron in composite models of quarks and leptons providing superficial violation of the PEP; the obtained upper limit on the electron size is $r_{0}<5.7 \times 10^{-18} \mathrm{~cm}$ (energy scale of $E \gtrsim 3.5 \mathrm{TeV}$ ).
\end{abstract}

PACS 29.40.Mc $95.30 . \mathrm{Cq}$

\section{Introduction}

The exclusion principle was postulated by W. Pauli in 1925 to explain atomic spectra and regularities of the Periodic Table of the elements. In modern Quantum Field Theory the

a e-mail: rita.bernabei@roma2.infn.it
Pauli Exclusion Principle (PEP) is related to the spin statistics [1] and automatically arises from the anti-commutation property of the fermion creation and destruction operators.

However-despite the fact that the foundation of PEP lies deep in the structure of Quantum Field Theory-a simple and easy explanation is still missing as nicely stressed by Feynman [2]. Thus, although all the well known successes of the PEP in explaining some atomic, condensed-matter and nuclear phenomena as well as the crucial role played in the discovery of quantum chromodynamics, the exact validity of the PEP is still an open question. In fact, the general principles of quantum theory do not require that all the particles must be either fermions or bosons, but also generalized statistics could be considered [3]. In particular, in the context of the many available theoretical attempts to go beyond Bose and Fermi statistics [4-8] it is useful to recall the "qcommutation algebra" developed in [9-11]:

$a_{i} a_{j}^{\dagger}-q a_{j}^{\dagger} a_{i}=\delta_{i j}, \quad-1 \leq q \leq 1$.

In the limit $q=-1+\beta^{2}$, with $\beta^{2} \ll 1$, this algebra can describe particles with a small probability $\left(\delta^{2}=\frac{\beta^{2}}{2}\right)$ of admixed symmetric component; thus the model provides small PEP violations.

Similar arguments have inspired many experimental tests of the PEP validity with improved sensitivities since the first pioneering experiments [12-15]. In particular, four classes of experiments have been considered so far: (i) searches for PEP-forbidden electronic states [16-19]; (ii) searches for PEP-forbidden nuclear states [20]; (iii) searches for PEPforbidden electronic transitions [21-24]; (iv) searches for PEP-forbidden nuclear transitions [25-29]. 
For completeness, it is worth noting that in 1980 Amado and Primakoff [30] criticized - on the basis of the assumption that the total Hamiltonian, describing the atoms, is completely symmetric in the electrons - the possibility of testing the Pauli principle by looking for PEP-forbidden transitions. However, their arguments can be evaded either as demonstrated in [31] (where apparently non-Paulian transitions could occur owing to the possible substructure of the electrons in composite models of quarks and leptons) or as suggested in [32] (where extra dimensions could lead to apparent PEP violations). Thus, experimental tests of PEPforbidden transitions can also investigate the deep structure of matter and/or of space-time. Moreover, in general, it is always wise to adopt the recommendation by Okun: "In fundamental physics if something could be tested it should be tested" [33].

\section{Results and discussions}

The results presented in the following have been obtained by analyzing data collected with the help of the DAMA/LIBRA set-up, whose description, radiopurity and main features are discussed in detail in the devoted [34]. The sensitive part of this set-up is made of 25 highly radiopure $\mathrm{NaI}(\mathrm{Tl})$ crystal scintillators (5-rows by 5 -columns matrix). ${ }^{1}$ Each $\mathrm{NaI}(\mathrm{Tl})$ detector has $9.70 \mathrm{~kg}$ mass and a size of $(10.2 \times 10.2 \times 25.4)$ $\mathrm{cm}^{3}$. The bare crystals are enveloped in Tetratec-teflon foils and encapsulated in radiopure OFHC Cu housing. In each detector two $10 \mathrm{~cm}$ long special quartz light guides act also as optical windows on the two end faces of the crystal and are coupled to two low background photomultipliers working in coincidence. The detectors are housed in a sealed low-radioactive copper box installed in the center of a low-radioactive $\mathrm{Cu} / \mathrm{Pb} / \mathrm{Cd}$-foils/polyethylene/paraffin shield; moreover, about $1 \mathrm{~m}$ concrete (made from the Gran Sasso rock material) almost fully surrounds (mostly outside the barrack) this passive shield, acting as a further neutron moderator. The copper box is maintained in HP nitrogen atmosphere in slightly overpressure with respect to the external environment; it is part of the threefold-levels sealing system which isolates the detectors from environmental air. A hardware/software system to monitor the running conditions is operative and self-controlled computer processes automatically control several parameters and manage alarms. For the radiopurity, the electronic chain, the data acquisition system and for all the other details, see [34].

The results, obtained in the investigation of possible nonPaulian emissions of protons with $\mathrm{E}_{p} \geq 10 \mathrm{MeV}$ in ${ }^{23} \mathrm{Na}$

\footnotetext{
${ }^{1}$ It is worth noting that an external detector of the second row from top was out of operation during the data taking discussed here because of a broken PMT (as also in [35]).
}

and in ${ }^{127} \mathrm{I}$ and of possible PEP-violating K-shell electron transitions in iodine atoms, are presented in the following. For this purpose the events, where just one detector fires, are considered (i.e. each detector has all the others as veto).

\subsection{Non-Paulian emissions of protons}

Since the nucleus is a fermion system, the nuclear structure is stable in case of an exact PEP, unless channels for strong, weak or electromagnetic decays are open. Thus, the usually considered stable nucleus would not a priori be absolutely stable in case small violations of the PEP would exist for a bound system of nucleons. In particular, some exotic transitions-normally forbidden by PEP in a stable nucleus-may occur. In this case (adopting a nuclear shell description) one of the nucleons in a higher energy shell may fall into a lower energy state, which is normally occupied; as a consequence (considering the relevant role of the nucleon-nucleon interaction), another nucleon can acquire enough energy to reach the unbound region and to escape from the nucleus.

The width of a single nucleon transition to an $i$ th occupied state, $\Gamma_{i}$, can be expressed according to $[25,26]$ as

$\Gamma_{i}=\delta_{i}^{2} \tilde{\Gamma}_{i}$

Here: (i) $\delta_{i}^{2}$ is the mixing probability of non-fermion statistics allowing for the transition to the occupied state $i$; (ii) $\tilde{\Gamma}_{i}$ is the width of the corresponding PEP-allowed transition whenever the final state $(i)$ would be empty.

In $[25,26]$ searches for non-Paulian single proton emission in $\mathrm{NaI}(\mathrm{Tl})$ have provided stringent limits for PEP validity in ${ }^{23} \mathrm{Na}$ and ${ }^{127} \mathrm{I}$. Here an analogous search for nonPaulian proton spontaneous emission with energy above $10 \mathrm{MeV}$ is performed by analyzing the data collected in a devoted running period of 23.7 days, when the data taking was optimized for the very high energy region. The presence of identified alphas from residual U/Th contamination has also offered references for the energy scale [34].

Figure 1 shows the 4-100 MeV energy distribution of the events measured by the 14 detectors in the three central rows of the DAMA/LIBRA detectors matrix (as already mentioned, another one in these rows was out of operation). No events are present above $10 \mathrm{MeV}$, and this directly gives an upper limit on the rate of non-Paulian processes leading to the emission of protons with energy above $10 \mathrm{MeV}$ equal to $(90 \%$ C.L.):

$$
\begin{aligned}
\lambda & =\lambda\left({ }^{23} \mathrm{Na}\right)+\lambda\left({ }^{127} \mathrm{I}\right) \leq 2.3(\epsilon N t)^{-1} \\
& =2.1 \times 10^{-33} \mathrm{~s}^{-1} .
\end{aligned}
$$

Here: (i) $N t=1.12 \times 10^{33}$ nuclei $\times \mathrm{s}$ is the exposure collected by the 14 detectors; (ii) $\epsilon$ is the proton detection efficiency (nearly 100\%); (iii) 2.3 is the limit at $90 \%$ C.L. when zero events are observed [36]. 


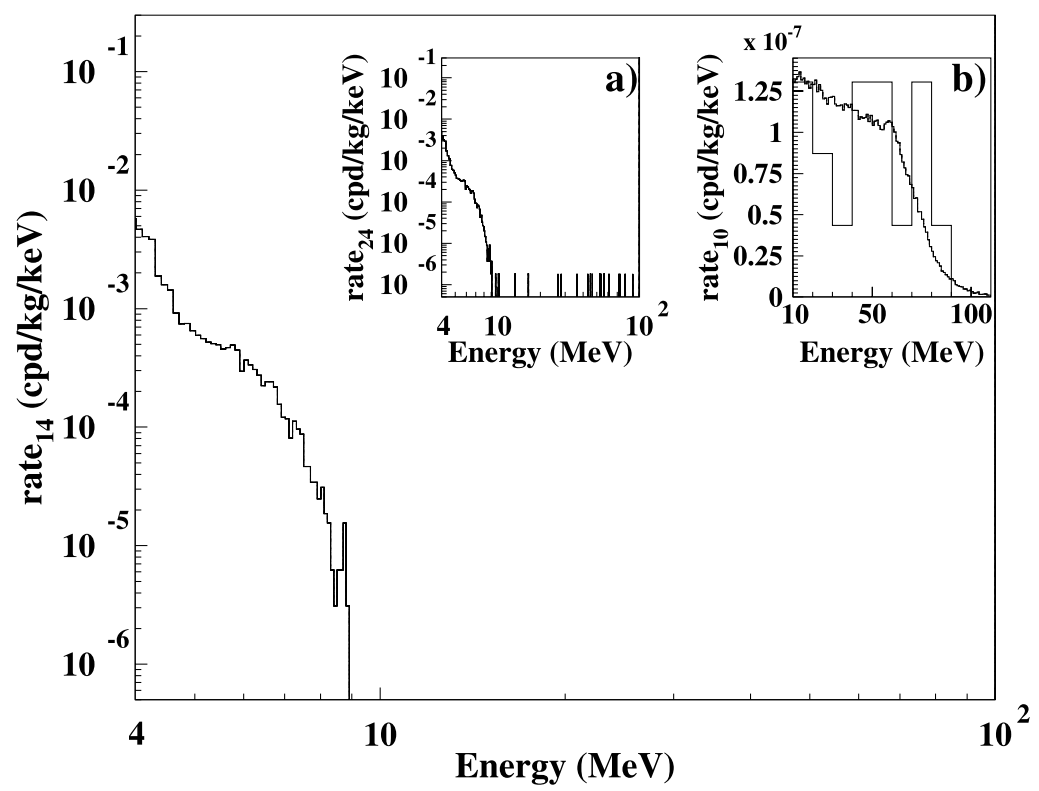

Fig. 1 Counting rate (rate 14 ) of the events measured by the 14 highly radiopure $\mathrm{NaI}(\mathrm{Tl})$ detectors in operation in the three central rows of the DAMA/LIBRA detectors matrix. The events in the 4-10 MeV energy region are essentially due to $\alpha$ particles from internal contaminants in the detectors (detailed studies are available in [34]). In inset (a) the counting rate measured by all the 24 working detectors ( rate $_{24}$ ) is shown. Events with $E>10 \mathrm{MeV}$ are present only in detectors be-

For completeness, in inset (a) is shown the measured energy distribution when considering all the 24 available detectors (that is, considering also the 5 detectors in the upper and the 5 in the lower rows of the detectors matrix); the total exposure is $1.9 \times 10^{33}$ nuclei $\times \mathrm{s}$. This figure shows that 17 events above $10 \mathrm{MeV}$ are instead cumulatively measured by the ten top and bottom detectors in the matrix; in particular, 11 of them are in the four detectors placed in the corners of the matrix. It is worth noting that the hypothesis that all these events might belong to PEP-violating proton emissions can be rejected with very high confidence level even by simple statistical considerations. In fact, assuming such an hypothesis (i.e. the expected number of events is the same for each detector), the expected number of events in 14 detectors-taking into account that 17 events are recorded by 10 detectors-should be $\simeq 14 \times \frac{17}{10}=23.8$; thus, the probability that instead 0 events are recorded by 14 detectors is: $e^{-23.8} \simeq 5 \times 10^{-11}$.

More in detail, in this very high energy region the background is due to the very high energy muons possibly surviving the mountain. Generally they give rise to events in which more than one detector fires, and thus they can be easily identified and are not competing background for the PEP-violating processes investigated here. Only muons impinging on the sensitive part of the set-up with a direction that forbids them to hit more than one detector can play a role. In order to have an accurate estimate of such a back- longing to the upper or to the lower rows in the detectors matrix. In inset (b) the same events as in (a) - with different binning — are shown above $10 \mathrm{MeV}$ (histogram) with superimposed a solid line, which corresponds to the background events expected from the vertical muon intensity distribution and the Gran Sasso rock overburden map of [37]. See text

ground, a suitable simulation has been realized on the basis of the set-up features, of the vertical muon intensity distribution and of the Gran Sasso rock overburden map of [37]. The simulation has shown that the observed 17 events (11 in the four corner detectors and 6 in the remaining 6 detectors in the upper and in the lower rows in the matrix) are fully consistent with the expectations for this kind of background; the results are summarized in Table 1 and compared with the experimental values. Furthermore, inset (b) of Fig. 1 shows the comparison between the measured energy distribution (histogram) and that expected from high energy muons able to survive the mountain (solid line) in those ten detectors, cumulatively. Finally, no events are present above $100 \mathrm{MeV}$ in whatever configuration, and this is consistent with the calculated expectations too.

In Table 1 the upper limits on the rates, $\lambda$, of non-Paulian processes leading to emission of protons with $E_{p} \geq 10 \mathrm{MeV}$ are also summarized for the various considered configurations. The quoted limits on $\lambda$ are calculated according to the distribution of a Poissonian process with background [36]:

$$
P_{J}(\lambda)=\frac{e^{-\left(b_{J}+s_{J}\right)} \sum_{k=0}^{n_{J}} \frac{\left(b_{J}+s_{J}\right)^{k}}{k !}}{e^{-b_{J}} \sum_{k=0}^{n_{J}} \frac{b_{J}^{k}}{k !}},
$$

where (i) the $J$ index identifies the considered group of detectors, (ii) $b_{J}$ is the expected background for the $J$ th group, (iii) $n_{J}$ is the number of measured events in the $J$ th group, 
Table 1 Comparison between the number of background events expected above $10 \mathrm{MeV}$ from high energy muons surviving the mountain and the number of measured events, considering various groups $(J)$ of detectors in the experimental set-up ( 5 rows by 5 columns detectors matrix, but an external detector out of operation); see text. Upper limits on the rates of non-Paulian processes leading to the emission of protons with $E_{p} \geq 10 \mathrm{MeV}$ are also reported for the various configurations; the final combined result is given

\begin{tabular}{|c|c|c|c|c|}
\hline $\begin{array}{l}\text { Group }(J) \text { of } \\
\text { considered } \\
\text { detectors }\end{array}$ & $\begin{array}{l}\text { Corresponding } \\
\text { exposure }\left(N_{J} t\right) \\
(\text { nuclei } \times \mathrm{s})\end{array}$ & $\begin{array}{l}\text { Expected } \\
\text { background } \\
\text { events }\left(b_{J}\right)\end{array}$ & $\begin{array}{l}\text { Measured } \\
\text { events }\left(n_{J}\right)\end{array}$ & $\begin{array}{l}\text { Upper limit on } \lambda \\
(90 \% \text { C.L.) } \\
\left(\mathrm{s}^{-1}\right)\end{array}$ \\
\hline $\begin{array}{l}\text { Just the } 4 \text { detectors } \\
\text { at corners (I) }\end{array}$ & $3.2 \times 10^{32}$ & 12.1 & 11 & $1.99 \times 10^{-32}$ \\
\hline Just the remaining 6 & & & & \\
\hline $\begin{array}{l}\text { detectors in the upper } \\
\text { and lower rows (II) }\end{array}$ & $4.8 \times 10^{32}$ & 8.7 & 6 & $9.33 \times 10^{-33}$ \\
\hline $\begin{array}{l}\text { Just the } 14 \text { central } \\
\text { detectors (III) }\end{array}$ & $1.1 \times 10^{33}$ & 2.2 & 0 & $2.06 \times 10^{-33}$ \\
\hline $\begin{array}{l}\text { Just the } 9 \text { core } \\
\text { detectors (IV) }\end{array}$ & $7.2 \times 10^{32}$ & 0.057 & 0 & $3.19 \times 10^{-33}$ \\
\hline Combined analysis $(\mathrm{I}+\mathrm{II}+\mathrm{III})$ : & & & & $1.63 \times 10^{-33}$ \\
\hline
\end{tabular}

(iv) $s_{J}=\lambda \epsilon N_{J} t$ is the expected PEP-violating transitions for the $J$ th group considering the collected exposure $N_{J} t$. The upper limit on $\lambda$ is obtained by the condition $P_{J}(\lambda)=\alpha$, where $\alpha=0.10$ for $90 \%$ C.L.

The final result from the 24 detectors is obtained by combining the independent groups I, II, III in Table 1; the cumulative probability is $P(\lambda)=\prod_{J} P_{J}(\lambda)$. From the final combined result given in Table $1, \lambda \leq 1.63 \times 10^{-33} \mathrm{~s}^{-1}$, one can derive the limit on the non-Paulian nuclear transition width:

$$
\begin{aligned}
\Gamma & =\Gamma\left({ }^{23} \mathrm{Na}\right)+\Gamma\left({ }^{127} \mathrm{I}\right)=\hbar \lambda \\
& \leq 1.1 \times 10^{-54} \mathrm{MeV}(90 \% \text { C.L. })
\end{aligned}
$$

This value represents an improvement of about a factor 3 with respect to the limits previously available. It is worth noting that a much larger sensitivity can be obtained by suitably increasing the devoted collected exposure, with the 9 inner core detectors, which have a high self-veto efficiency.

From the previous value-considering the same nuclear physics frameworks as in [26] —one can derive the average probability, $\delta^{2}$, which quantifies the possible PEP violations. In fact, the total nuclear width can be written as

$\tilde{\Gamma}=\tilde{\Gamma}\left({ }^{23} \mathrm{Na}\right)+\tilde{\Gamma}\left({ }^{127} \mathrm{I}\right)=\Gamma / \delta^{2}$.

The $\tilde{\Gamma}$ have been calculated for ${ }^{23} \mathrm{Na}$ and ${ }^{127} \mathrm{I}$ according to two possible models for the momentum distribution functions of the nucleons in the bound state: (a) a Fermi momentum distribution with $k_{F}=255 \mathrm{MeV} / c$; (b) "realistic" functions taking into account the correlation effects. In the latter case, the distribution function calculated for ${ }^{56} \mathrm{Fe}$ has been adopted both for ${ }^{23} \mathrm{Na}$ and for ${ }^{127} \mathrm{I}$, owing to the fact
Table 2 Expected values of $\tilde{\Gamma}$ for ${ }^{23} \mathrm{Na}$ and ${ }^{127} \mathrm{I}$; the proton energy threshold is $E_{p}=10 \mathrm{MeV}$. Case (a) refers to a Fermi momentum distribution with $k_{F}=255 \mathrm{MeV} / c$, while in case (b) the momentum distribution of ${ }^{56} \mathrm{Fe}$ accounting also for the correlation effects (see [26] for details) has been adopted. The relative upper limits on $\delta^{2}$ are given

\begin{tabular}{llcl}
\hline Case & ${ }^{A} X$ & $\begin{array}{l}\tilde{\Gamma} \\
(\mathrm{MeV})\end{array}$ & $\begin{array}{l}\delta^{2} \text { Upper limit } \\
(90 \% \text { C.L. })\end{array}$ \\
\hline (a) & ${ }^{23} \mathrm{Na}$ & 1.65 & $1.7 \times 10^{-55}$ \\
& ${ }^{127} \mathrm{I}$ & 4.64 & \\
(b) & ${ }^{23} \mathrm{Na}$ & 4.59 & $6.8 \times 10^{-56}$ \\
& ${ }^{127} \mathrm{I}$ & 11.1 & \\
\hline
\end{tabular}

that all these functions are quite similar for all the nuclei with $A \geq 12$.

The $\tilde{\Gamma}$ widths expected for ${ }^{23} \mathrm{Na}$ and ${ }^{127} \mathrm{I}$ are given in Table 2 according to the two models, considered here, and for a proton energy threshold of $E_{p}=10 \mathrm{MeV}$ (see [26] for the details of the calculation). Considering that these values are clearly model dependent, a cautious conclusion can be adopted on the limit estimated for $\delta^{2}: \delta^{2} \lesssim 3-4 \times 10^{-55}$.

Finally, considering a $\tilde{\Gamma}$ energy behavior as in [26], one can roughly estimate a lower limit on the mean life for nonPaulian proton emission. In particular, in the framework of case (b) one obtains $\tau_{\mathrm{Na}} \gtrsim 2 \times 10^{25} \mathrm{yr}$ and $\tau_{\mathrm{I}} \gtrsim 2.5 \times 10^{25} \mathrm{yr}$. These limits improve those previously available for ${ }^{23} \mathrm{Na}$ and ${ }^{127} \mathrm{I}[25,26]$.

\subsection{PEP-violating electron transitions in iodine atom}

In this subsection the case of PEP-violating electron transitions in atoms is investigated. For this purpose, in the follow- 
ing we analyze the data collected by DAMA/LIBRA [34] in the low energy range of interest here; the exposure is 0.53 ton $\times$ yr. It is worth noting that in this case the available exposure is very large since the set-up usually takes data in a condition optimized for the low energy region because of other kinds of investigations [35].

Since the electron atomic transitions of sodium atoms are below the experimental energy threshold, we analyze here just the case of electron atomic transitions of iodine atoms.

Following a PEP-violating electronic transition, X-rays and Auger electrons can be emitted due to both the transition itself and to the subsequent atomic shell rearrangements; at these energies large $\mathrm{NaI}(\mathrm{Tl})$ detectors-as those of DAMA/LIBRA - practically collect all the energies (detection efficiency $\sim 1$ ). The total energy release is expected to be at the level of the ionization energy for the considered shell; however, it actually is slightly lower (by a term $\Delta$ ), because of the presence of the other electrons in the already filled shells. In case of detectors with a very sharp energy resolution, the precise knowledge of the small energy shift between the PEP-violating transitions and the PEPallowed ones can be exploited in order to disentangle them. This has been pursued e.g. by the VIP experiment [23, 24], which has a very good energy resolution at the energy of the examined PEP-violating transition $(\simeq 8 \mathrm{keV}): \sigma / E \sim$ $1.7 \%$. In that case the shift between the PEP-violating and the PEP-allowed transitions was calculated by the DiracFock method with an associated uncertainty $<10 \mathrm{eV}$. In the present case, considering the shielding due to the extra electron filling the final shell, the energy of PEP-violating transitions in iodine $(Z=53)$ should be similar to the relative PEP-allowed transitions in tellurium $(Z=52)$ and, thus, in the case of the $\mathrm{K}$ shell $\Delta \lesssim 1.5 \mathrm{keV}$. This value has to be compared with the energy resolution: $\sigma \sim 3 \mathrm{keV}$ at the energy of the iodine $\mathrm{K}$ shell [34], i.e. in the present case $\Delta$ is well below the energy resolution of the used detectors; therefore, precise estimations by the Dirac-Fock method are unnecessary. Thus, the energy of $32 \mathrm{keV}$ is considered for the $\mathrm{K}$-shell PEP-violating transition.

In Fig. 2 the energy distribution of the events is shown in the low energy range of interest here. As can be observed, no evidence for the expected peak is present there. Thus, the number of events which can be ascribed-at a give C.L.- to the process searched for has been preliminarily estimated by using the so-called "one $\sigma$ approach": the maximum number of events which can be hidden by background fluctuations at one sigma level is estimated for the considered decay mode as the square root of the number of background events in the given energy window. In spite of its simplicity, this method gives the right scale of the sensitivity of the experiment.

In the considered case of iodine K-shell PEP-violating process, the searched peak is at $32 \mathrm{keV}$ energy and in the

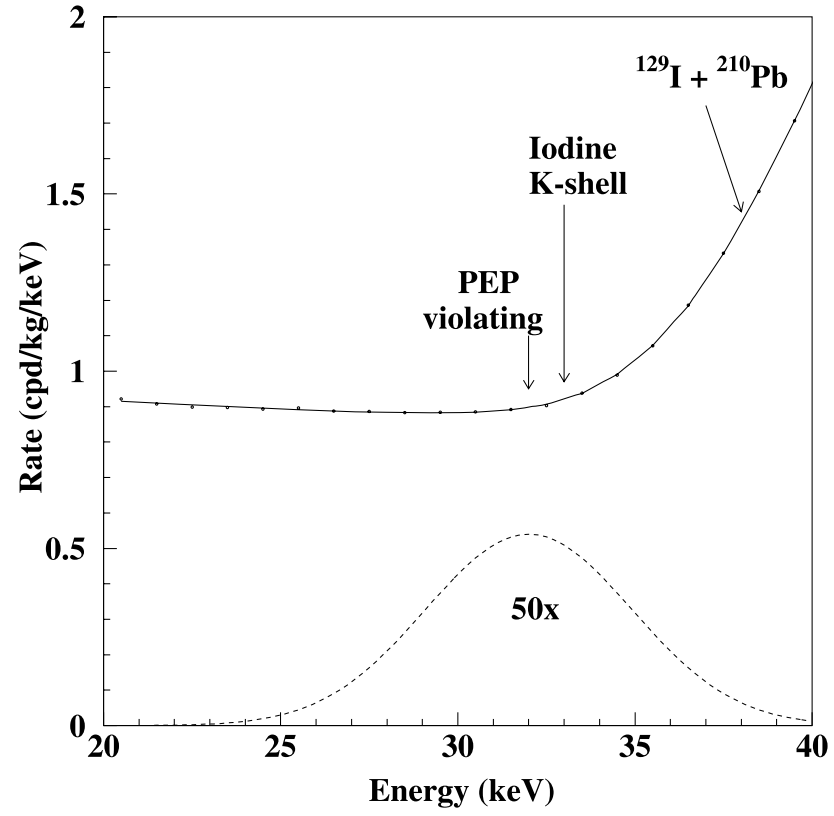

Fig. 2 Energy distribution of the events measured by DAMA/LIBRA [34] in the region of interest for the PEP-violating K-shell transitions; the exposure is here 0.53 ton $\times$ yr. The line represents the result of the fit described in the text. The Gaussian is 50 times the signal excluded by the present analysis at $90 \%$ C.L.

$\pm 1 \sigma$ window around it $(2 \sigma=5.66 \mathrm{keV})$ the maximum number of events, which can be hidden by background fluctuations, is $8.3 \times 10^{-3} \mathrm{cpd} / \mathrm{kg}$ (90\% C.L.). Thus, from this value the limit on the number of PEP-violating K-shell transitions in iodine is derived to be $1.2 \times 10^{-2} \mathrm{cpd} / \mathrm{kg}$ (90\% C.L.).

Then, the data have been analyzed by fitting them with a simple background model (a linear behavior and the contribution from the presence of ${ }^{129} \mathrm{I}$ and ${ }^{210} \mathrm{~Pb}$ estimated according to the measurements discussed in [34]), and the possible PEP-violating contribution from the $\mathrm{K}$ shell (here represented as a Gaussian centered at $32 \mathrm{keV}$ with $\sigma=2.83 \mathrm{keV}$ and area $S$ ). Thus, in the calculation 3 free parameters ( 2 for the linear behavior and $S$ for the Gaussian) have been used and the degrees of freedom were $(22-3)=19$. In particular, the procedure has been performed minimizing the $\chi^{2}$ function by the MINUIT package [38]; the profile of the $\chi^{2}$ function was well symmetric. The $\chi^{2}$ of the best fit was about 30. In this way the contribution from PEP-violating transitions has been obtained to be $S=(0.038 \pm 0.022) \mathrm{cpd} / \mathrm{kg}$, which corresponds to an upper limit of $S<0.074 \mathrm{cpd} / \mathrm{kg}$ (90\% C.L.) following the Feldman and Cousins procedure [39]. It worth noting that on slightly moving the Gaussian peak position $( \pm 1 \mathrm{keV})$ similar or more stringent values are obtained.

Since $4.01 \times 10^{24}$ iodine atoms are present in one $\mathrm{kg}$ of $\mathrm{NaI}(\mathrm{Tl})$, the previous limit implies that lifetimes $\left(\tau_{\mathrm{PV}}\right)$ shorter than $4.7 \times 10^{30} \mathrm{~s}$ can be excluded at $90 \%$ C.L. It should be noted that the $\tau_{\mathrm{PV}}$ is related to the lifetime $\left(\tau^{0}\right)$ of 
the PEP-allowed transition of outer electrons into a free $\mathrm{K}$ shell when an hole is present: $\tau^{0}=\delta_{e}^{2} \tau_{\mathrm{PV}} ; \delta_{e}^{2}$ is the probability of the admixed symmetric component for the electron [21].

The normal electromagnetic dipole transition to the iodine $\mathrm{K}$ shell is typically $\tau^{0} \sim 6 \times 10^{-17} \mathrm{~s}$ [15]; therefore, the limit $\delta_{e}^{2}<1.28 \times 10^{-47}(90 \%$ C.L.) holds. It is worth noting that the achieved limit is one order of magnitude more stringent than the one by ELEGANTS V [21] and that the sensitivity reached by the VIP experiment [23, 24] for the $\mathrm{Cu}$ electronic transition is of the order of $10^{-28}$, with a final goal at level of $10^{-31}$. $^{2}$

In [31] superficial violations of the PEP due to the possible substructure of electrons have been discussed in composite models of quarks and leptons. In this theory the possible finite size of the electron, $r_{0}$, is related to $\delta_{e}^{2}$ by the relation [31]:

$\delta_{e}^{2}=\left[\frac{4}{3}\left(\frac{3}{7}\right)^{5}\left(\frac{Z r_{0}}{a_{0}}\right)^{3}\right]^{2}$,

where $a_{0}$ is the Bohr radius $\left(=0.53 \times 10^{-8} \mathrm{~cm}\right)$ and $Z$ is the atomic number. Thus, the upper limit on $\delta_{e}^{2}$ obtained above implies an upper limit on the electron size: $r_{0}<$ $5.7 \times 10^{-18} \mathrm{~cm}$; this value corresponds to an energy scale of $E \gtrsim 3.5 \mathrm{TeV}$.

\section{Conclusions}

The highly radiopure DAMA/LIBRA set-up is in operation at the Gran Sasso National Laboratory of the INFN; it has a sensitive mass of about $250 \mathrm{~kg}$ of highly radiopure $\mathrm{NaI}(\mathrm{Tl})$. By means of this set-up new searches for non-Paulian nuclear processes, i.e. processes normally forbidden by the Pauli exclusion principle, have been carried out.

In particular, a new improved limit for the spontaneous emission rate of protons with energy $E_{p} \gtrsim 10 \mathrm{MeV}$ has been derived to be $1.63 \times 10^{-33} \mathrm{~s}^{-1}$. The corresponding limit on the relative strength for the searched non-Paulian transition is $\delta^{2} \lesssim(3-4) \times 10^{-55}$. Moreover, PEP-violating electron transitions in iodine atoms have also been investi- gated, excluding lifetimes shorter than $4.7 \times 10^{30} \mathrm{~s}$ at $90 \%$ C.L. and the limit $\delta_{e}^{2}<1.28 \times 10^{-47}$ (90\% C.L.) is derived. This latter limit can also be related to a possible finite size of the electron in composite models of quarks and leptons, providing superficial violation of the PEP; the obtained upper limit on the electron size is $r_{0}<5.7 \times 10^{-18} \mathrm{~cm}$ (energy scale of $E \gtrsim 3.5 \mathrm{TeV}$ ).

\section{References}

1. W. Pauli, Phys. Rev. 58, 716 (1940)

2. R.P. Feynman, R.B. Leighton, M. Sands, The Feynman Lectures on Physics, vol. 3 (Addison-Wesley, Reading, 1963)

3. A.M. Messiah, O.W. Greenberg, Phys. Rev. B 136, 248 (1964)

4. G. Gentile, Nuovo Cimento 17, 493 (1940)

5. H.S. Green, Phys. Rev. 90, 270 (1953)

6. A.Yu. Ignatiev, V.A. Kuzmin, Sov. J. Nucl. Phys. 461, 786 (1987)

7. V.N. Gavrin, A.Yu. Ignatiev, V.A. Kuzmin, Phys. Lett. B 206, 343 (1988)

8. O.W. Greenberg, R.N. Mohapatra, Phys. Rev. Lett. 59, 2507 (1987)

9. O.W. Greenberg, Phys. Rev. Lett. 64, 705 (1990)

10. R.N. Mohapatra, Phys. Lett. B 242, 407 (1990)

11. O.W. Greenberg, R.C. Hilborn, Fund. Phys. 29, 397 (1999)

12. M. Goldhaber, G. Goldhaber, Phys. Rev. 73, 1472 (1948)

13. E. Fischbach, T. Kirsten, O.A. Schaeffer, Phys. Rev. Lett. 20, 1012 (1968)

14. B.A. Logan, A. Ljubicic, Phys. Rev. C 20, 1957 (1979)

15. F. Reines, H.W. Sobel, Phys. Rev. Lett. 23, 954 (1974)

16. V.M. Novikov et al., Phys. Lett. B 240, 227 (1990)

17. K. Deilamian et al., Phys. Rev. Lett. 74, 4787 (1995)

18. A.S. Barabash et al., JETP Lett. 68, 112 (1998)

19. D. Javorsek II et al., Phys. Rev. Lett. 85, 2701 (2000)

20. E. Nolte et al., J. Phys. G 17, S355 (1991)

21. H. Ejiri et al., Nucl. Phys. B (Proc. Suppl.) 28A, 219 (1992)

22. P. Belli et al., Phys. Lett. B 460, 236 (1999)

23. S. Bartalucci et al., Phys. Lett. B 641, 18 (2006)

24. E. Milotti et al., Int. J. Mod. Phys. A 22, 242 (2007)

25. H. Ejiri, H. Toki, Phys. Lett. B 306, 218 (1993)

26. R. Bernabei et al., Phys. Lett. B 408, 439 (1997)

27. R. Arnold et al., Eur. Phys. J. A 6, 361 (1999)

28. R. Arnold et al., Nucl. Phys. B (Proc. Suppl.) 87, 510 (2000)

29. H.O. Back et al., Eur. Phys. J. C 37, 421 (2004)

30. R.D. Amado, H. Primakoff, Phys. Rev. C 22, 1338 (1980)

31. K. Akama et al., Phys. Rev. Lett. 68, 1826 (1992)

32. O.W. Greenberg, R.N. Mohapatra, Phys. Rev. D 39, 2032 (1989)

33. L.B. Okun, Comments Nucl. Part. Phys. 19, 99 (1989)

34. R. Bernabei et al., Nucl. Instrum. Methods A 592, 297 (2008)

35. R. Bernabei et al., Eur. Phys. J. C 56, 333 (2008)

36. Review of Particle Properties, Phys. Rev. D 45, III.40 (1992)

37. M. Ambrosio et al., Phys. Rev. D 52, 3793 (1995)

38. CN/ASD Group, MINUIT user guide, nProgram Library D506 CERN (1993)

39. G.J. Feldman, R.D. Cousins, Phys. Rev. D 57, 3873 (1998)

\footnotetext{
${ }^{2}$ Let us note that the VIP experiment exploits a different approach to overcome the argument of [30] (see Sect. 1). In fact, the PEP-violating effect in VIP is proportional to the number of electrons that pass through the conductor and to the number of scattering processes on the atoms of the $\mathrm{Cu}$ lattice.
} 